\title{
Influence of elastic scattering on the measurement of core-level binding energy dispersion in X-ray photoemission spectroscopy
}

\author{
E.F. Schwier ${ }^{1, a}$, C. Monney ${ }^{2}$, N. Mariotti ${ }^{1}$, Z. Vydrovà ${ }^{1}$, M. García-Fernández ${ }^{1}$, C. $\operatorname{Didiot}^{1}$, \\ M.G. Garnier ${ }^{1}$, and P. Aebi ${ }^{1}$ \\ 1 Département de Physique and Fribourg Center for Nanomaterials, Université de Fribourg, 1700 Fribourg, Switzerland \\ 2 Research Department Synchrotron Radiation and Nanotechnology, Paul Scherrer Institute, 5232 Villigen PSI, Switzerland
}

Received 20 January 2011 / Received in final form 9 May 2011

Published online 22 June 2011 - (c) EDP Sciences, Società Italiana di Fisica, Springer-Verlag 2011

\begin{abstract}
We explore the interplay between the elastic scattering of photoelectrons and the surface core level shifts with regard to the determination of core level binding energies in $\mathrm{Au}(111)$ and $\mathrm{Cu}_{3} \mathrm{Au}(100)$. We find that an artificial shift is created in the binding energies of the Au $4 f$ core levels, that exhibits a dependence on the emission angle, as well as on the spectral intensity of the core level emission itself. Using a simple model, we are able to reproduce the angular dependence of the shift and relate it to the anisotropy in the electron emission from the bulk layers. Our results demonstrate that interpretation of variation of the binding energy of core-levels should be conducted with great care and must take into account the possible influence of artificial shifts induced by elastic scattering.
\end{abstract}

\section{Introduction}

The general assumption of non dispersing core levels is only valid for fully localized states, but due to the continuous nature of the electronic wave function, the orbitals of core levels can exhibit weak hybridization even for binding energies of up to a few hundred $\mathrm{eV}$. This has been demonstrated for gaseous molecules like $\mathrm{C}_{2} \mathrm{H}_{2}$ [1] and $\mathrm{N}_{2}[2]$ and in recent measurements on graphene by Lizzit et al. [3] who were able to determine the bandwidth of the $\mathrm{C} 1 \mathrm{~s}$ core level to be $60 \mathrm{meV}$. The corresponding theoretical prediction from ab initio calculations support the claim, that these orbitals are not completely degenerate.

To resolve the dispersion of core levels and the size of the Brillouin zone in solids with X-ray photoelectron spectroscopy (XPS), high angular and energy resolutions are necessary. In addition to that, there exist two mechanisms that introduce a broadening into the measurement. These are the quasi elastic scattering of the photoelectron with the atoms of the crystal [4,5] as well as the influence of phonons on the photoemission process $[6,7]$. A third factor which has not been considered until now and that can introduce a systematic error to the measurement of the dispersion in core-level binding energies is introduced in this paper with the demonstration of an angular dependent artificial shift, created by the existence of unresolved energetically shifted surface core levels.

The surface core level shift $\left(\Delta E_{S C L S}\right)$ describes the energy shift between the core levels attributed to the bulk

\footnotetext{
a e-mail: eike.schwier@unifr.ch
}

and the surface of a crystal [8]. It arises from a combination of initial [9] and final state [10] effects. The non continuous charge distribution and the reduced number of neighboring atoms at the surface of the crystal can change the coulomb potential in the topmost layers with respect to its bulk value, leading to a shift in the core level binding energies of the surface atoms. In addition to this, the screening of the photohole can be different at the surface or in the bulk, also influencing the surface core level shift. Measurements on noble metal films [11], $5 d$ metals [12] graphite [13] and rare-earth crystals [14] have shown that the displacement between bulk and surface core levels can be positive as well as negative and possesses an amplitude of up to several hundred meV.

It is important to note that at kinetic energies above $500 \mathrm{eV}$ the strong anisotropy in the individual electronatom scattering leads to a focusing of electron flux along directions pointing from the emitting atom to the scatterer (Fig. 1a) [15]. This effect is also known as X-ray photoelectron diffraction (XPD). In $2 \pi$ angular XPD patterns (Fig. 1b) high symmetry directions as well as low index lattice planes can be identified through forward focusing peaks and Kikuchi lines. Both structures are used to provide local information about the atomic structure near the surface [16-18].

In this work we focus on the influence of the elastic scattering of photoelectrons on the binding energy of core levels. We demonstrate a correlation between the binding energy and the emission intensity of the core level and propose a mechanism that explains the angular dependence of core level binding energies. 


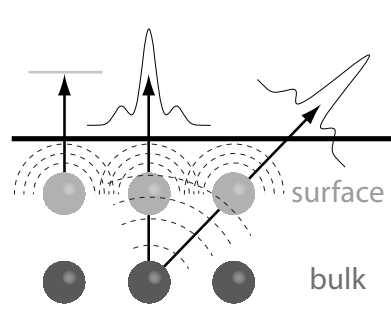

a)

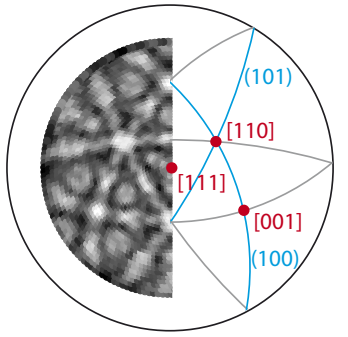

b)
Fig. 1. (Color online) (a) Contribution to the scattering amplitude from the surface atoms (light grey) and the bulk atoms (dark grey). Closed packed directions in the bulk change the scattering amplitude for different emission angles. The contribution from the surface layer remains almost constant up to grazing emission. (b) A typical $2 \pi$ angular scan in stereographic projection with intensity anisotropy due to scattering of photoelectrons. The high symmetry directions are visible as forward focusing peaks (points), while high symmetry planes in the bulk manifest themselves as Kikuchi bands (lines). Normal emission is plotted at the center while the black circle corresponds to emission at grazing angles. Maximum and minimum intensity corresponds to white and black, respectively.

\section{Experiment}

All measurements were performed with an upgraded SCIENTA SES 200 analyzer, allowing for multiple angle parallel detection, using a non-monochromatized $\operatorname{MgK}_{\alpha}(h \nu=$ $1253.6 \mathrm{eV}$ ) X-ray anode as excitation source. A computer controlled 5-axis manipulator allows rotations of the sample along the polar and azimuthal directions with a precision of $0.1^{\circ}$ and $0.2^{\circ}$, respectively. The angular acceptance of the entrance hole was $2.4^{\circ}$. The energy steps of the spectra were set to $19 \mathrm{meV}$ for the $2 \pi$ angular scans and to $50 \mathrm{meV}$ for the other measurements. All spectra were taken at room temperature with parallel detection in angle and energy. During the measurement, the pressure in the chamber did not exceed $2 \times 10^{-10} \mathrm{mbar}$. The crystals were prepared with multiple sputter and annealing cycles. The sputtering acceleration voltage was set to $1.5 \mathrm{kV}$ and the incident angle of the argon ions was chosen to be $65^{\circ}$ off normal while the sample was rotated.

To determine the influence of the elastic scattering of photoelectrons on the binding energy of core levels, we have chosen the $\mathrm{Au} 4 f$ doublet of the $\mathrm{Au}(111)$ and $\mathrm{Cu}_{3} \mathrm{Au}(100)$ surfaces, as they exhibit a relatively large surface core-level shift $\left(\mathrm{Au}(111): \Delta E_{S C L S}=0.35 \pm\right.$ $0.01 \mathrm{eV}[19]$ and in the case of $\mathrm{Cu}_{3} \mathrm{Au}(100): \Delta E_{S C L S}=$ $0.5 \pm 0.05 \mathrm{eV}$ from the works of DiCenzo et al. [20] and $\left.\Delta E_{S C L S}=0.41 \pm 0.01 \mathrm{eV}[21]\right)$. Also, the surface preparation and properties of the $\mathrm{Au}(111)$ herringbone reconstruction $[22,23]$ as well as the $\mathrm{Cu}_{3} \mathrm{Au}(100)-c(2 \times 2)$ surface reconstruction [24-26] are well documented.

The annealing temperature of the Au crystal was set to $600{ }^{\circ} \mathrm{C}$ in order to obtain the herringbone surface reconstruction. The $\mathrm{Cu}_{3} \mathrm{Au}$ was heated up to $450{ }^{\circ} \mathrm{C}$ and cooled down within several hours across the transition temperature $\left(T_{C}=390{ }^{\circ} \mathrm{C}\right)$ to obtain the $c(2 \times 2)$ reconstruction. The pressure never exceeded $8 \times 10^{-10}$ mbar. After each

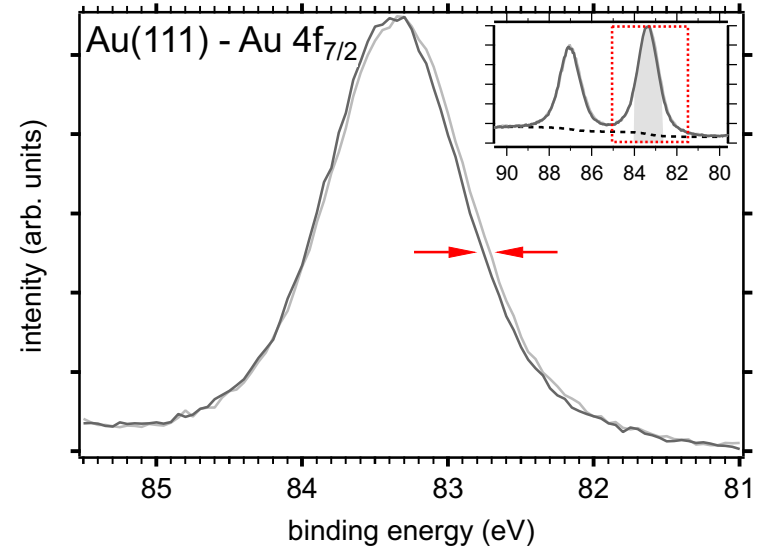

Fig. 2. (Color online) Two spectra of the Au $4 f_{7 / 2}$ core level of $\mathrm{Au}(111)$, taken at $68^{\circ}$ off normal at azimuthal angles corresponding to the [001] symmetry direction (dark grey) and $12^{\circ}$ off symmetry (light grey). A shift of $40 \mathrm{meV}$ (arrows) is visible. Both spectra are normalized to the maximum intensity of the $\mathrm{Au} 4 f_{7 / 2}$ peak. Inset: the whole Au $4 f$ doublet with Shirley background. The grey area below the peak corresponds to the interval used for the general fitting procedures (see text).

preparation cycle, the surface order and cleanliness were tested with LEED and XPS measurements, respectively.

\section{Results}

Two spectra (Fig. 2) of the $\mathrm{Au} 4 f$ doublet on $\mathrm{Au}(111$ ) were taken at the same polar angle, but at two different azimuthal angles. While one was measured in a direction that coincides with a low index crystal direction, the other spectrum was measured $12^{\circ}$ away from that symmetry point. The energy broadening of the $\mathrm{Au} 4 f$ peak allows to approximate its shape with a Gaussian profile including a constant background. Fitting the two Au $4 f_{7 / 2}$ peaks with this profile gives a shift of $\Delta E_{B}=40 \pm 5 \mathrm{meV}$ between the binding energy of the two spectra. Considering the relatively high electron energies and the broadening inherent to the XPS experiment here, it is clear, that the shift cannot by attributed to a dispersion induced by weakly hybridized $\mathrm{Au} 4 f$ core levels.

To determine the angular dependence of the shift in the spectra of $\mathrm{Au}(111)$, a $2 \pi$ solid angle emission scan of the Au $4 f$ doublet was measured. Therefor the intensity of the electron emission was recorded as a function of the polar angle $\Theta$ and the azimuthal angle $\Phi$, as well as the the core level binding energy $E_{B}$ generating a set of energy resolved spectra of the core level emission as a function of the electron emission angles. In addition to that a polar angle was chosen, where a high resolution azimuthal scan was performed. The resulting spectra $I\left(E_{B}, \Theta, \Phi\right)$ were fitted in energy with a Gaussian profile, including a constant background. To test the stability of the fit, an increased fitting interval as well as a fixed width for the Gaussian profile were implemented into the fit without changing the quality of the results. In addition, the same behavior was found for the Au $4 f_{5 / 2}$ peak or upon using a Voigt profile 


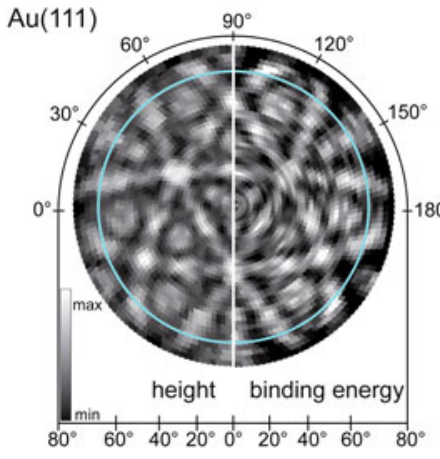

a)

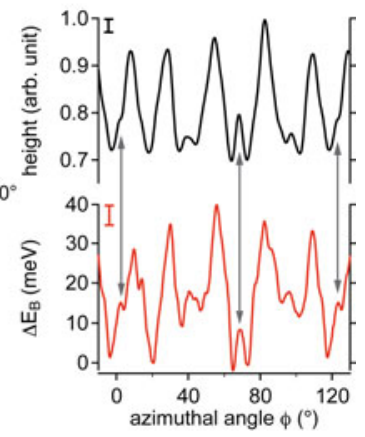

b)
Fig. 3. (Color online) (a) Stereographic projection of the angular dependence of the height (left half) and binding energy (right half) of the Gaussian profile used to fit the $\mathrm{Au} 4 f_{7 / 2}$ peak of $\mathrm{Au}(111)$. The position of the azimuthal cut is marked in blue (b) Plot of the height $h$ (black) and binding energy $E_{B}$ (red) of the $\mathrm{Au} 4 f_{7 / 2}$ peak from $\mathrm{Au}(111)$ as a function of azimuthal angle, taken at $\Theta=68^{\circ}$. Grey arrows mark corresponding fine structures. The error bars from the fitting parameters are in the top left of the azimuthal plots.

with a nonzero Lorenzian component or a Shirley type [27] background.

To increase the contrast of the anisotropy in the peak height $h(\Theta, \Phi)$ and the binding energy shift $\Delta E_{B}(\Theta, \Phi)$ of the $\mathrm{Au} 4 f_{7 / 2}$ peak a smooth background was subtracted from each dataset. The resulting diffraction pattern are plotted in a stereographic plot (Fig. 3a). Comparison of the height and binding energy shows that the main features in the scattering anisotropy, i.e., the forward focusing peaks as well as the Kikuchi lines, are clearly visible in both height and binding energy. Even fine structures of the anisotropy are replicated in the binding energy as it can be seen in the data from the high resolution azimuthal scan (Fig. 3b, arrows). As all of these features correspond to electron diffraction induced by the bulk ordering of low index closed packed crystal directions and planes a bulk mediated effect can be proposed.

The binding energy of the $\mathrm{Au} 4 f_{7 / 2}$ exhibits a decrease of several $100 \mathrm{meV}$ in the untreated data while changing the polar angle from normal to grazing emission. This shift is proportional to $1 / \cos \Theta$ and can be interpreted as an increased ratio between the emission from surface and bulk core levels caused by the finite electron mean free path. Even though this variation is only dependent on the polar angle, it suggests that the surface core level shift plays a role in the original observed shift shown in Figure 2. To support this hypothesis, the $\mathrm{Cu}_{3} \mathrm{Au}(100)$ alloy was chosen for further measurement, as it exhibits a larger surface core level shift and should therefore exhibit a larger amplitude in $\Delta E_{B}$ during the azimuthal scans.

The same $2 \pi$ angular scan and a corresponding high resolution azimuthal scan were performed for the $\mathrm{Cu}_{3} \mathrm{Au}$ compound. The correlation between the peak height $h(\Theta, \Phi)$ and the binding energy $E_{B}(\Theta, \Phi)$ of the $\mathrm{Au} 4 f_{7 / 2}$ peak (Figs. 4a and $4 \mathrm{~b}$ ) is similar to the results found for $\mathrm{Au}(111)$. A comparison between the maximum amplitude

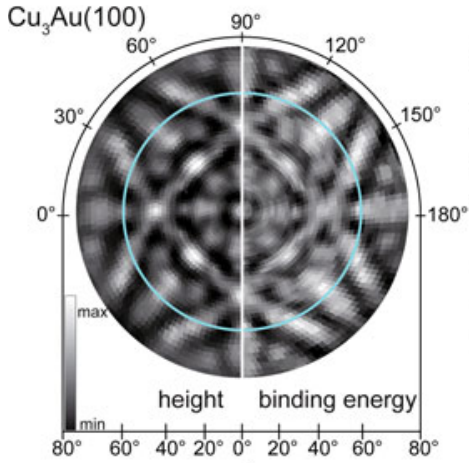

a)

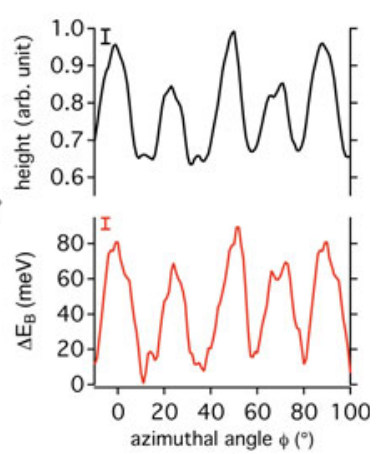

b)
Fig. 4. (Color online) (a) As Figure 3a for $\mathrm{Cu}_{3} \mathrm{Au}(100)$. (b) As Figure $3 \mathrm{~b}$ for $\mathrm{Cu}_{3} \mathrm{Au}(100)$ with $\Theta=58^{\circ}$.

of the shift $\Delta E_{B}$ for $\mathrm{Au}(111)\left(\Delta E_{B} \approx 40 \mathrm{meV}\right.$, Fig. 3b) and $\mathrm{Cu}_{3} \mathrm{Au}(100)\left(\Delta E_{B} \approx 90 \mathrm{meV}\right.$, Fig. 4b) supports the proposed influence of the size of the surface core level shift on the amplitude of the angular dependence in the binding energy shift.

\section{Model}

This dependence of the binding energy on the emission angle can be understood if the different scattering amplitudes between photoelectrons from the surface and bulk components are considered. We propose a simple model (Fig. 5a), that by considering the $\mathrm{Au} 4 f_{7 / 2}$ peak as a sum of a surface and a bulk component, can describe the measured azimuthal dependence of $\Delta E_{B}$. The model peak consists of two Gaussian peaks with identical width $(\sigma=1.3 \mathrm{eV})$, corresponding to our experimental broadening. We assume that the measured anisotropy in the azimuthal scan (Fig. 4b) is caused entirely by the angular dependence of the scattering of the bulk component. The mean surface to bulk ratio $(S / B=0.55)$ for an emission angle of $\Theta=58^{\circ}$ on $\mathrm{Cu}_{3} \mathrm{Au}$, was calculated from the results of DiCenzo et al. [20]. To account for the uncertainty of the binding energy separation between the surface and the bulk component, two sets of fits were performed using values for $\triangle E_{S C L S}$ corresponding to the upper $\left(\Delta E_{S C L S}=0.55 \mathrm{eV}[20]\right)$ and lower $\left(\Delta E_{S C L S}=0.40 \mathrm{eV}[21]\right)$ limit of the literature values for the surface core level shift of $\mathrm{Cu}_{3} \mathrm{Au}$. The variation in the fit between the two values is visualized through a confidence band in the plot.

The resulting composite peak was fitted with a Gaussian profile, in the same manner as the measured data and the binding energy shift was plotted as a function of the azimuthal angle. In Figure 5b (top) the dependence of the calculated binding energy (grey band) is compared to the experimental results (red). The model predicts a shift $\Delta E_{B}$, which is larger than the experimental results. This can be corrected by relaxing the oversimplified assumption of a Gaussian profile with a constant peak width. Note also that, using a Voigt Profile instead of a Gaussian 


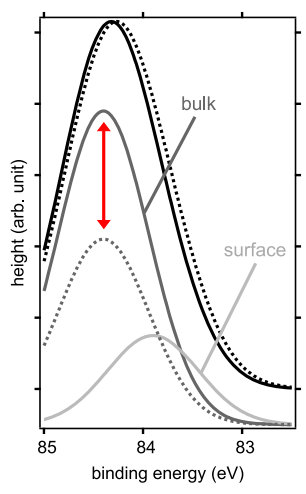

a)

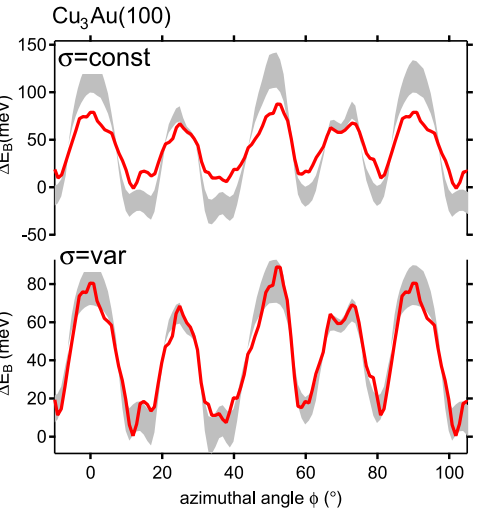

b)
Fig. 5. (Color online) (a) Peak composition considered for the model. The bulk peak (dark grey) changes its height (dotted line) as a function of azimuthal angle, while the surface contribution (light grey) stays constant. The composite peak (black) exhibits an apparent shift (dotted line). (b) Comparison of the measured binding energy shift $\Delta E_{B}$ (red) with the confidence bands (see text) from a model peak composition assuming a constant (grey, top) and variable (grey, bottom) bulk peak width.

does not change the overestimation of the binding energy shift.

A good agreement between the model and the experiment can be achieved if the width of the Gaussian peak corresponding to the bulk emission is allowed to vary (Fig. 5b, bottom). A possible mechanism that would lead to a variation of the peak width is the increase of the information depth through electron diffraction. Along directions were forward focusing takes place, the electrons contributing to a core level spectra will on average be emitted from a greater depth and travel a longer path through the solid, thus increasing the inelastic losses of the photo electrons. Another possible source for a peak broadening is the change in cross section for scattering events with the atoms in the closed packed directions, leading to an increase in quasi elastic losses.

In a first order approximation, the broadening is assumed to scale linearly with the intensity variation of the bulk peak. This can be reasoned by comparing the information depth with the emitted intensity, which both scale with the mean free path. The gain in the intensity that is created through the forward focusing, will increase the mean depth from where electrons are emitted and therefore increase the possibility of occurrences of processes that involve energy loss. This approximation is still applicable, if the defocusing of photoelectrons through multiple scattering events in forward focusing directions [28-30] is taken into account, as it effectively introduces a scaling factor to the intensity emitted in forward focusing directions.

If a linear dependence of the bulk peak width on the bulk anisotropy is implemented into the model $(\sigma(\Phi)=$ $\sigma_{0} h(\Phi) / h_{0}$, with $\sigma_{0}$ and $h_{0}$ being the average peak width and height, respectively) and the resulting composite peak is again fitted in the same way as described above, the calculated confidence band for the binding energy shift (Fig. 5b (bottom)) coincides with the results from the experiment.

\section{Discussion}

Although the present experiments have been conducted with quite moderate resolution $\left(\mathrm{MgK}_{\alpha}\right)$ and on model systems $\left(\mathrm{Au}\right.$ and $\mathrm{Cu}_{3} \mathrm{Au}$ ), the findings are also relevant for higher resolution experiments where core level shifts are smaller and remain unresolved.

To illustrate the influence of this effect on experiments that probe the dispersion of core levels the same calculation as in the model above, can be performed for peaks with a linewidth of $\sigma=300 \mathrm{meV}$ and a surface core level shift of $\Delta E_{S C L S}=50 \mathrm{meV}$, representing a high resolution measurement with a monochromatic excitation source and an unresolved core level. Using the same anisotropy in the electron emission as for $\mathrm{Cu}_{3} \mathrm{Au}(100)$ the amplitude of $\Delta E_{B}$ would be approximately $10 \mathrm{meV}$, leading to a non negligible systematic error in the measurement of the core level dispersion.

In the absence of a surface core level shift, a shift $\Delta E_{B}$ will nonetheless be present if at least two energetically separated lattice sites for the same element exist and if the coordination of those sites inside the lattice leads to distinct electron scattering patterns. In that case, the anisotropic electron emission and the correlation between the intensity of the core level emission and its binding energy would be more complicated however.

Even in the case of measurements on systems as simple as monolayers, the emission from two differently coordinated sites within the layer (e.g., C $1 s$ in graphene with partial $\mathrm{H}$ adsorption [31]) should lead to a shift through multiple scattering within the layer or with the substrate atoms. However, as the scattering amplitude of such processes is usually small compared to bulk mediated scattering, the strength of such shifts is expected to be less pronounced.

\section{Conclusion}

We have demonstrated that the emission angle dependent shifts in the binding energy of the $\mathrm{Au} 4 f_{7 / 2}$ core level, that were observed on $\mathrm{Au}(111)$ and $\mathrm{Cu}_{3} \mathrm{Au}(100)$ surfaces, can be explained by a simple mechanism based on a peak composition that includes an energetically unresolved surface core level. Our model uses the surface core level shift, the intensity ratio between the surface and bulk emission, the overall peak broadening and the diffraction of photoelectrons emitted from bulk lattice sites. It is able to explain the shift in binding energy between measurements at different emission angles and its correlation to the emission intensity.

The measured shifts are qualitatively described by a model that assumes a constant width for the bulk contribution. However, if a dependence of the peak width to the 
anisotropy of the emission is introduced, the experimental results and the calculated values match within the error range.

The effect that has been described in this paper could be used to identify the presence of unresolved core level shifts. More interesting could be the possibility to use the amplitude of the shift and its variation upon surface changes as an additional probe in the analysis of surfaces.

This project was supported by the Fonds National Suisse pour la Recherche Scientifique through Division II and the Swiss National Center of Competence in Research MaNEP.

We would also like to thank the mechanical workshops in Neuchâtel and Fribourg for their support.

\section{References}

1. B. Kempgens, H. Köppel, A. Kivimäki, M. Neeb, L. Cederbaum, A. Bradshaw, Phys. Rev. Lett. 79, 3617 (1997)

2. U. Hergenhahn, O. Kugeler, A. Rüdel, E. Rennie, A. Bradshaw, J. Phys. Chem. A 105, 5704 (2001)

3. S. Lizzit, G. Zampieri, L. Petaccia, R. Larciprete, P. Lacovig, E. Rienks, G. Bihlmayer, A. Baraldi, P. Hofmann, Nature Phys. 6, 345 (2010)

4. H. Boersch, R. Wolter, H. Schoenebeck, Z. Phys. 199, 349 (1967)

5. N. Erickson, C. Powell, Phys. Rev. B 40, 7284 (1989)

6. R. White, C.S. Fadley, M. Sagurton, Z. Hussain, Phys. Rev. B 34, 5226 (1986)

7. L. Plucinski, J. Minár, B. Sell, J. Braun, H. Ebert, C. Schneider, C.S. Fadley, Phys. Rev. B 78, 35108 (2008)

8. J. Houston, R. Park, G. Laramore, Phys. Rev. Lett. 30 , 846 (1973)

9. B. Johansson, N. Mårtensson, Phys. Rev. B 21, 4427 (1980)
10. E. Pehlke, M. Scheffler, Phys. Rev. Lett. 71, 2338 (1993)

11. P. Citrin, G. Wertheim, Y. Baer, Phys. Rev. Lett. 41, 1425 (1978)

12. N. Mårtensson, H. Saalfeld, H. Kuhlenbeck, M. Neumann, Phys. Rev. B 39, 8181 (1989)

13. K. Prince, I. Ulrych, M. Peloi, B. Ressel, V. Chab, C. Crotti, C. Comicioli, Phys. Rev. B 62, 6866 (2000)

14. N. Shamsutdinov, W. Sloof, A. Böttger, J. Appl. Phys. 98, 014908 (2005)

15. K. Siegbahn, U. Gelius, H. Siegbahn, E. Olson, Phys. Scripta 1, 272 (1970)

16. C.S. Fadley, Phys. Scripta 1987, 39 (1987)

17. S.A. Chambers, Adv. Phys. 40, 357 (1991)

18. R. Fasel, P. Aebi, J. Osterwalder, L. Schlapbach, Surf. Sci. 331, 80 (1995)

19. P. Heimann, J.V. der Veen, D. Eastman, Solid State Commun. 38, 595 (1981)

20. S. DiCenzo, P. Citrin, E.H. Jr, G.W. Jr, Phys. Rev. B 34, 1343 (1986)

21. W. Eberhardt, S. Wu, R. Garrett, D. Sondericker, F. Jona, Phys. Rev. B 31, 8285 (1985)

22. J. Barth, H. Brune, G. Ertl, R. Behm, Phys. Rev. B 42, 9307 (1990)

23. F. Reinert, G. Nicolay, Appl. Physics A 78, 817 (2004)

24. T. Buck, G. Wheatley, L. Marchut, Phys. Rev. Lett. 51, $43(1983)$

25. A. Stuck, J. Osterwalder, T. Greber, S. Hüfner, L. Schlapbach, Phys. Rev. Lett. 65, 3029 (1990)

26. H. Dosch, L. Mailänder, H. Reichert, J. Peisl, R. Johnson, Phys. Rev. B 43, 13172 (1991)

27. D. Shirley, Phys. Rev. B 5, 4709 (1972)

28. S. Tong, H. Poon, D. Snider, Phys. Rev. B 32, 2096 (1985)

29. W.E. Egelhoff, Phys. Rev. Lett. 59, 559 (1987)

30. H. Aebischer, T. Greber, J. Osterwalder, A. Kaduwela, D. Friedman, G. Herman, C.S. Fadley, Surf. Sci. 239, 261 (1990)

31. R. Balog, B. Jørgensen, L. Nilsson, M. Andersen, E. Rienks, M. Bianchi, M. Fanetti, E. Lægsgaard, A. Baraldi, S. Lizzit et al., Nat. Mater. 9, 315 (2010) 